\title{
The multiphysics modeling of heat and moisture induced stress and strain of historic building materials and artefacts
}

\author{
Natalie Williams Portal' ( $(\varangle)$, A.W.M. van Schijndel ${ }^{2}$, Angela Sasic Kalagasidis' \\ 1. Department of Civil and Environmental Engineering, Chalmers University of Technology, Sven Hultins Gata 8, Göteborg, Sweden \\ 2. Department of Building Physics and Services, Eindhoven University of Technology, 5600MB Eindhoven, the Netherlands
}

\begin{abstract}
The basic structure of historic sites and their associated interior artefacts can be damaged or even destroyed by climate change. The evaluation of combined heat and moisture induced stress and strain (HMSS) can predict possible damage-related processes. In this paper, the development of one- and two-dimensional HMSS models of building materials and artefacts in COMSOL Multiphysics Version 4, a commercial finite element software, is presented. The validation of the numerical models is revealed using analytical, numerical and experimental solutions. As a result, the HMSS model was shown to be an adequate predictive tool to determine possible damage-related processes in building assemblies and artefacts.
\end{abstract}

\author{
Keywords \\ heat, \\ moisture, \\ mechanical stress, \\ multiphysics modelling, \\ predictive tools, \\ historical buildings \\ Article History \\ Received: 16 October 2012 \\ Revised: 6 May 2013 \\ Accepted: 5 August 2013 \\ () Tsinghua University Press and \\ Springer-Verlag Berlin Heidelberg \\ 2013
}

\section{Research aims}

The aim of this paper is to present a two-dimensional numerical model that can be used to study coupled heat and moisture induced stresses and strains in materials and objects, which include artefacts that are exposed to varying hygrothermal loads in indoor and outdoor environments.

\section{Introduction}

Thermal and moisture durability of materials and items inside a building is one of the main research topics in building physics science. Numerical models and tools that have been developed for that purpose considerably help in understanding deterioration and degradation processes that take place in buildings. Advanced building physics tools, which are capable of combined thermal and mass (i.e. water, air, salt) analyses in porous media, are required for durability studies (Hagentoft 1998; Nicolai et al. 2007; Sevilgen and Kilic 2011; Woloszyn and Rode 2008). However, the structural performance of a material is typically not included in the building physics tools even though hygrothermal loads have been shown to induce stresses and strains in porous media in (Mihoubi and Bellagi 2009; Kowalski and Smoczkiewicz-Wojciechowska 2007; Kowalkski and Rybicki 2004). For instance, displacements, dimensional change or cracking are typical issues associated with wooden structural elements exposed to indoor and outdoor hygrothermal loads. These induced changes, in turn, modify the mechanical properties of the given media and as such alter its longterm behaviour. The implementation of a multidisciplinary approach can lead to a more comprehensive understanding, analysis and prevention of hygrothermal-induced problems in buildings (Burke 2009).

Numerical tools from the structural engineering research field, though offering advanced non-linear finite element analyses of the structural performance of a material, are often of limited applicability in building physics investigations. Structural tools typically provide options to prescribe constant, simplified or even uncoupled moisture and thermal

E-mail: Natalie.williams@chalmers.se 


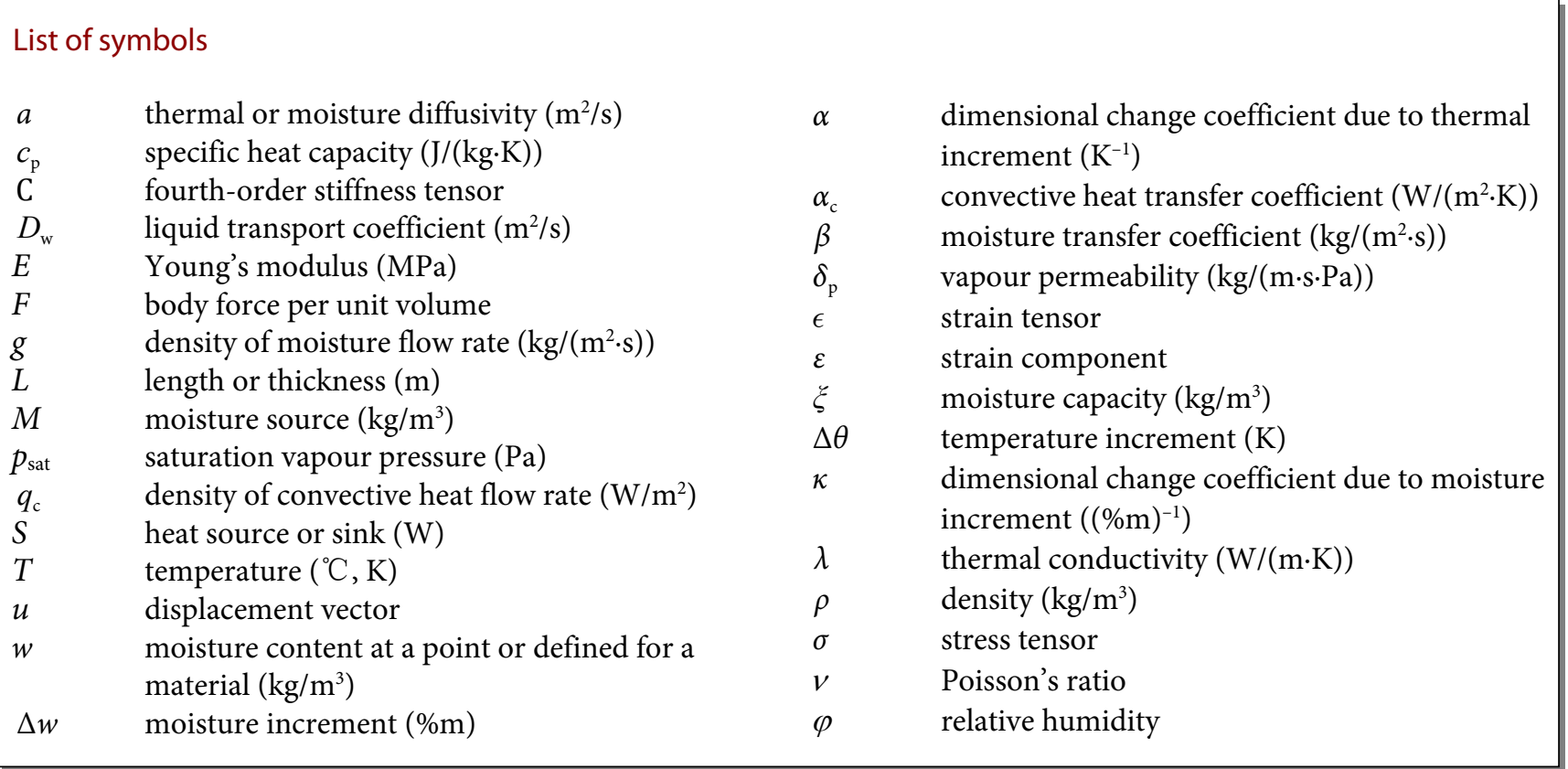

loads. As demonstrated in (Mirianon et al. 2008), an external script was required by a structural tool to incorporate varying humidity and structural loading as well as moisturedependent wood properties, while maintaining isothermal conditions. Other attempts to quantify hygrothermalinduced mechanical response of materials include similar mathematically or experimentally fitted models (Castellini et al. 2003; Dionisi Vici et al. 2006; Jakieła et al. 2008; Bratasza et al. 2012 Lukomski 2012). This separate representation of hygrothermal response of a material is an insufficient presentation of thermal and moisture loads from the imposed environment. Despite current limitations, computational research has shown promising results and the development of numerical models has been deemed as valuable for predicting past and future behaviour of materials. For the purpose of in-depth analysis of durability of materials like wood, there is a large interest in models and tools that could be used for combined hygrothermal and structural analyses.

When studying a combined hygrothermal and structural performance of a material, both the magnitude and the historical pattern of the hygrothermal loads play a role. Noticeable deviations from normal working conditions should always be carefully treated as they may complicate the existing durability issues or may even enforce new ones. Large research efforts are currently focused on predicting the hygrothermal performance and durability of the building materials with respect to the possible climate changes. The Climate for Culture project within the European Union's 7th framework program attempts to face the challenges of climate change by establishing attainable environmental guidelines for museums while acknowledging the need for the preservation of cultural heritage. As of late, there has not only been a push to reduce energy consumption and carbon emissions, but also a concern regarding the preservation of natural resources and cultural heritage according to ICA (2010). Therefore, it has become of interest to gain knowledge of the past and present conditions of artefacts in order to understand causes of previous damage, predict forthcoming damage, and also aid in developing preservation guidelines for artefacts. Numerical simulations are shown to be the only method which can yield the desired knowledge of the future performance of materials.

This paper presents a study built upon the research by Williams Portal (2011) encompassing the development of one - and two-dimensional coupled heat and moisture induced stress and strain (HMSS) models in COMSOL software package (COMSOL 2010). The verification of these models is executed in various stages by means of analytical, numerical and experimental solutions. It is worth mentioning that the availability of verification tools for coupled hygrothermal and structural numerical models such as HMSS is limited. Within conservatory, however, there are case studies which are relevant to this type of modeling and one in particular (Rachwał et al. 2010), is used for the experimental validation of HMSS. The numerical simulation results for a case study consisting of an artefact's relation to a building assembly in a historic building are also included. In addition, the sensitivity of the model to additional moisture sources (i.e. rain) is evaluated. General conclusions and recommendations about the applicability of the HMSS models are presented. 


\section{Heat and moisture induced mechanical stress and strain modeling}

This study primarily focuses on the development of a general model that can be adapted to simulate HMSS of various hygroscopic materials found in historical buildings subjected to variable indoor and outdoor climates. The HMSS model was developed in two stages based on the COMSOL numerical modeling by Schellen (2011). COMSOL is a finite element analysis software providing the flexibility to model and simulate multiple physics in one environment (COMSOL 2010). Firstly, a one-dimensional transient coupled heat and moisture (HM) model was generated and verified to ensure the numerical validity of the simulation results (see Section 4). This verified model was expanded to include the computation of the mechanical stresses and strains which are induced by the coupled thermal and hygric transport. As a result of this coupling, numerical simulations for temperature and moisture profiles, as well as resulting mechanical stresses and strains in a defined hygroscopic material can be obtained. A flow chart illustrated in Fig. 1 describes the coupling of the HM and HMSS models.

\subsection{Heat and moisture transfer}

The model is based on two-dimensional conductive heat transfer according to Fourier's Law with a moisture dependent thermal conductivity:

$q_{\mathrm{c}}=-\lambda \nabla T=-\left(\lambda \frac{\partial T}{\partial x}, \lambda \frac{\partial T}{\partial y}\right)$

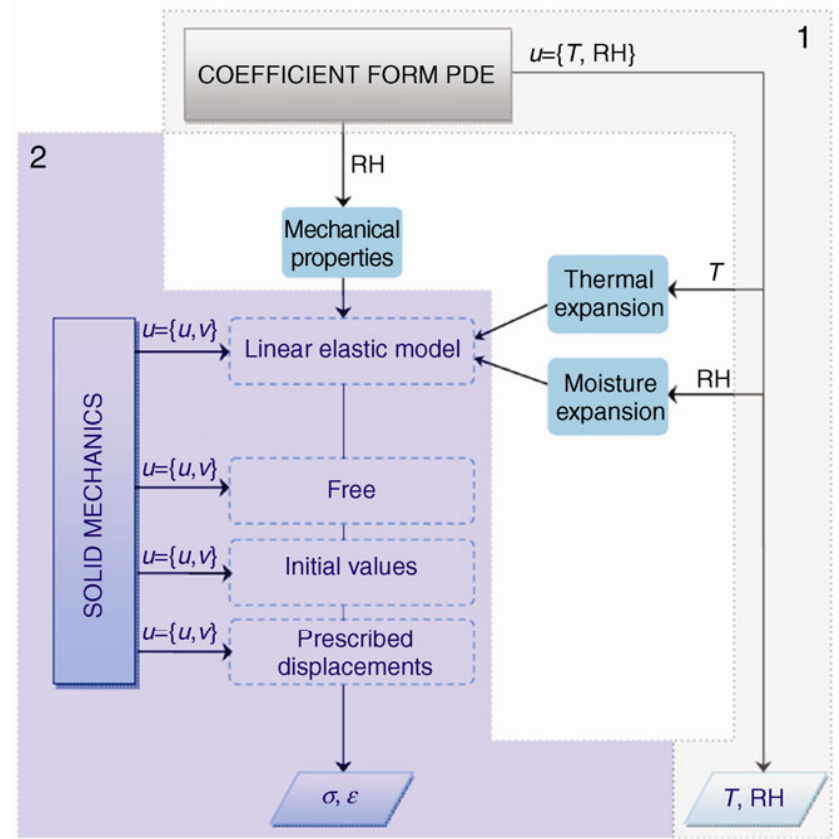

Fig. 1 Flow chart illustrating the coupling of the HM (Zone 1) and HMSS (Zones 1 \& 2) models
The total moisture transfer considered includes both twodimensional vapour and liquid flow, as described respectively in Eqs. (2) to (4).

$g=g_{\mathrm{v}}+g_{\mathrm{L}}$

$g_{\mathrm{v}}=-\delta_{\mathrm{p}} \cdot p_{\mathrm{sat}} \nabla \varphi=-\delta_{\mathrm{p}} \cdot p_{\mathrm{sat}}\left(\frac{\partial \varphi}{\partial x}, \frac{\partial \varphi}{\partial y}\right)$

$g_{\mathrm{L}}=-D_{\mathrm{w}} \cdot \xi \nabla \varphi=-D_{w} \cdot \xi\left(\frac{\partial \varphi}{\partial x}, \frac{\partial \varphi}{\partial y}\right)$

The moisture state can be characterized by several different potentials, namely moisture content $\left[\mathrm{kg} / \mathrm{m}^{3}\right]$, partial vapour pressure [Pa], relative humidity [\%] (Hagentoft 2002), and suction pressure $[\mathrm{Pa}]$. In this study, moisture transfer was initially characterized by both partial vapour pressure and relative humidity. Through model verification, however, it was shown that partial vapour pressure as a potential poses limitations particularly under non-isothermal conditions with a simultaneous step change in hygric conditions. A delayed response in the convergence of the prescribed boundary step change in both temperature and partial vapour pressure was observed in the numerical simulation results. In short, it is thought that this delayed response is caused by the fact that the heat front propagates considerably faster than the moisture front (i.e. with a factor of $\left.5 \times 10^{4}\right)$. Moreover, the relative humidity was observed to be an effective potential in this particular case. A prescribed step change in relative humidity is in fact a function of temperature, therefore being directly correlated to the non-isothermal conditions. The logarithmic of suction pressure (LPc) is also considered to be a suitable moisture potential particularly for extreme boundary conditions such as non-isothermal or intensive heat and moisture sources (i.e. solar radiation and rain). The LPc potential, however, was not further considered here, as it is rather computationally demanding in ordinary HM type modeling (van Schijndel 2011).

\subsection{Energy and moisture balance equations}

The coupling of dynamic heat and moisture transports is accomplished by means of the partial differential equations (PDE) for energy and moisture balance. These relationships can be expressed by Eqs. (5) and (6):

$c_{\mathrm{p}} \rho \frac{\partial T}{\partial t}=-\nabla(-\lambda \nabla T) \pm S$

$\xi \frac{\partial \varphi}{\partial t}=-\nabla\left(\left(-\delta_{\mathrm{p}} \cdot p_{\mathrm{sat}}-D_{\mathrm{w}} \cdot \xi\right) \nabla \varphi\right) \pm M$ 
It is of particular interest in this study to observe the correlation between temperature and relative humidity, denoted as dependent variables $T$ and $\varphi$ respectively. These two variables, along with the associated coefficients are assembled into vectors and matrices:

$$
\left[\begin{array}{cc}
\rho c_{\mathrm{p}} & 0 \\
0 & \xi
\end{array}\right]\left[\begin{array}{l}
\frac{\partial T}{\partial t} \\
\frac{\partial \varphi}{\partial t}
\end{array}\right]+\left[\begin{array}{cc}
\lambda & 0 \\
0 & \delta_{\mathrm{p}} \cdot p_{\text {sat }}+D_{\mathrm{w}} \cdot \xi
\end{array}\right]\left[\begin{array}{l}
\nabla^{2} T \\
\nabla^{2} \varphi
\end{array}\right] \pm\left[\begin{array}{l}
S \\
M
\end{array}\right]
$$

The effect of temperature on the vapour diffusion (i.e. thermal diffusion) was found to be negligible in this study (see Section 4.1) and as such is set to zero in Eq. (7). This assumption is in accordance with the findings in (Janssen 2011) that showed basically the absence of thermal diffusion for the most common building materials. Even where thermal diffusion could be experimentally detected, like in wood (Segerholm 2007), it was shown that the effect of thermal diffusion could be aptly combined with a main vapour transport parameter rather than described by a stand-alone term, which is composed of a temperature gradient and a corresponding transfer coefficient (Janssen 2011; Segerholm 2007).

Moreover, in order to formulate a boundary value problem using Eq. (7), two natural boundary conditions are defined:

$q_{\mathrm{c}}=\alpha_{\mathrm{c}} \cdot\left(T_{\mathrm{s}}-T_{\mathrm{a}}\right)$

$g=\beta \cdot\left(\varphi_{\mathrm{s}}-\varphi_{\mathrm{a}}\right)$

These prescribed convective thermal and hygric fluxes describe the interaction between the inside of the domain (subscript s) with the exterior conditions (subscript a). The thermal and moisture transfer coefficients are assumed to be constant for interior environments and are calculated according to the wind speed for exterior environments. In the case of heat and moisture sources at a surface, such as sun irradiation or rain, both equations can be extended by respective source terms. An example is presented in Section 5.4 , where a sensitivity analysis regarding rain load on a wall is provided.

\subsection{Mechanical stress and strain}

The HMSS model was developed as a linear elastic boundary value problem. This type of problem is based on equilibrium, compatibility and constitutive relationships of a threedimensional volume element in a continuous body (Eschenauer 1997). The mechanical equilibrium is based on three tensor partial differential equations that can be summarized by the equation of motion such that con- ditions of static equilibrium are considered:

$\nabla \sigma+F=0$

Compatibility is expressed by six equations describing the small strain-displacement of a continuous body. The total strain tensor is written in the simplified form as

$\epsilon=\frac{1}{2}\left[\nabla u+\nabla u^{\mathrm{T}}\right]$

The constitutive relationships are based on the linear proportionality between stress and strain tensors as described by Hooke's law:

$\sigma=\mathrm{C}: \epsilon$

The Voigt notation can be used to express the above relationship in matrix form, shown for a two-dimensional isotropic problem:

$\left[\begin{array}{l}\sigma_{x x} \\ \sigma_{y y} \\ \sigma_{x y}\end{array}\right]=\left[\begin{array}{lll}C_{11} & C_{12} & C_{13} \\ C_{21} & C_{22} & C_{23} \\ C_{31} & C_{32} & C_{33}\end{array}\right]\left[\begin{array}{l}\varepsilon_{x x} \\ \varepsilon_{y y} \\ \varepsilon_{x y}\end{array}\right]$

The C-matrix can be further expressed by means of two independent material coefficients, namely Young's modulus, $E$, and Poisson's ratio, v (Ottosen and Petersson 1992). This matrix is shown in Eq. (14) for a plane strain problem. In the HMSS model, Young's modulus is a function of moisture content and Poisson's ratio is a constant coefficient.

$\mathrm{C}=\frac{E}{(1+v)(1-2 v)}\left[\begin{array}{ccc}1-v & v & 0 \\ v & 1-v & 0 \\ 0 & 0 & \frac{(1-2 v)}{2}\end{array}\right]$

The Hooke-Duhamel's law for thermo-elastic materials can be used to relate the stress and strain tensors, along with temperature and moisture. In this study, the displacement formulation is used, as it is of interest to solve for unknown displacements as per the following:

$\sigma=\sigma_{0}+\mathrm{C}:\left(\epsilon-\epsilon_{0}-\alpha \Delta \theta-k \Delta w\right)$

The inclusion of the thermal and moisture strain deformations in the strain tensor, according to Eq. (15), allows for the coupling of the HM and HMSS models. The dimensional change coefficients included in the model are constants. The boundary value problem using the displacement formulation requires prescribed displacements as boundary conditions. The assigned boundary values are variable depending on the geometry and orientation of the domain taken in consideration. 


\section{Model verification and validation}

Verification of the HM and HMSS models were executed in various stages in order to ensure the numerical validity of the simulation results. The one-dimensional stationary and transient decoupled heat and moisture transfer mechanisms were primarily verified in (Williams Portal 2011; Williams Portal et al. 2011) using an analytical solution presented in (Hagentoft 2011). In addition, one-dimensional transient coupled heat and moisture transfer was verified by means of the European Provisional Standard prEN 15026 (2004). Two-dimensional transient HMSS modeling was verified using experimental and numerical simulation results derived from a study by Rachwał et al. (2010) which evaluated the response of lime wood supports in historical panel paintings to climatic changes.

\subsection{Normative benchmark of the HM model}

The coupled model was verified with the normative benchmark test of European Provisional Standard prEN 15026 (2004). The benchmark test is based on an analytical solution for the coupled thermal and hygric transport in a homogeneous semi-infinite domain that is initially in equilibrium with constant surrounding conditions of $T=20^{\circ} \mathrm{C}$ and $\varphi=50 \%$. The domain is thereafter exposed to a step change to $T=30^{\circ} \mathrm{C}$ and $\varphi=95 \%$. For the purpose of finding the analytical solution for this problem, boundary resistances and moisture sources are neglected in this benchmark (Claesson 2002). In reality, boundary conditions exist and it is important to include them in modeling as further demonstrated in Section 5. Lastly, temperature and moisture profiles after 7, 30 and 365 days are to be calculated by the model. These profile results are required to fall within $\pm 2.5 \%$ of the analytical solution. Hygrothermal material properties are specified by analytical functions in the prEN 15026 benchmark (2004) and are used in the model verification.

Numerical simulation results for temperature and water content profiles after 7, 30 and 365 days were found to be within the limits required by the benchmark, see Fig. 2 . This model appears to be a valid predictive tool to investigate the impact of variable thermal and hygric conditions on building components and artefacts found in historical buildings.

\subsection{Numerical and experimental verification of the HMSS model}

As mentioned in Section 2, experimental and numerical studies that can be used for the verification of coupled models, such as HMSS, are rare. The most appropriate references are typically found within conservatory. Accordingly, the
HMSS model was verified using experimental and numerical simulated results presented in one study by Rachwał et al. (2010). This study evaluates the response of lime wood supports in historical panel paintings subjected to changing climate conditions particularly related to moisture. The historical use of this material is exemplified in Fig. 3 (Dardes and Rothe 1995).

One of the various tests executed in the study by Rachwał et al. (2010) is the tangential dimensional response of a lime
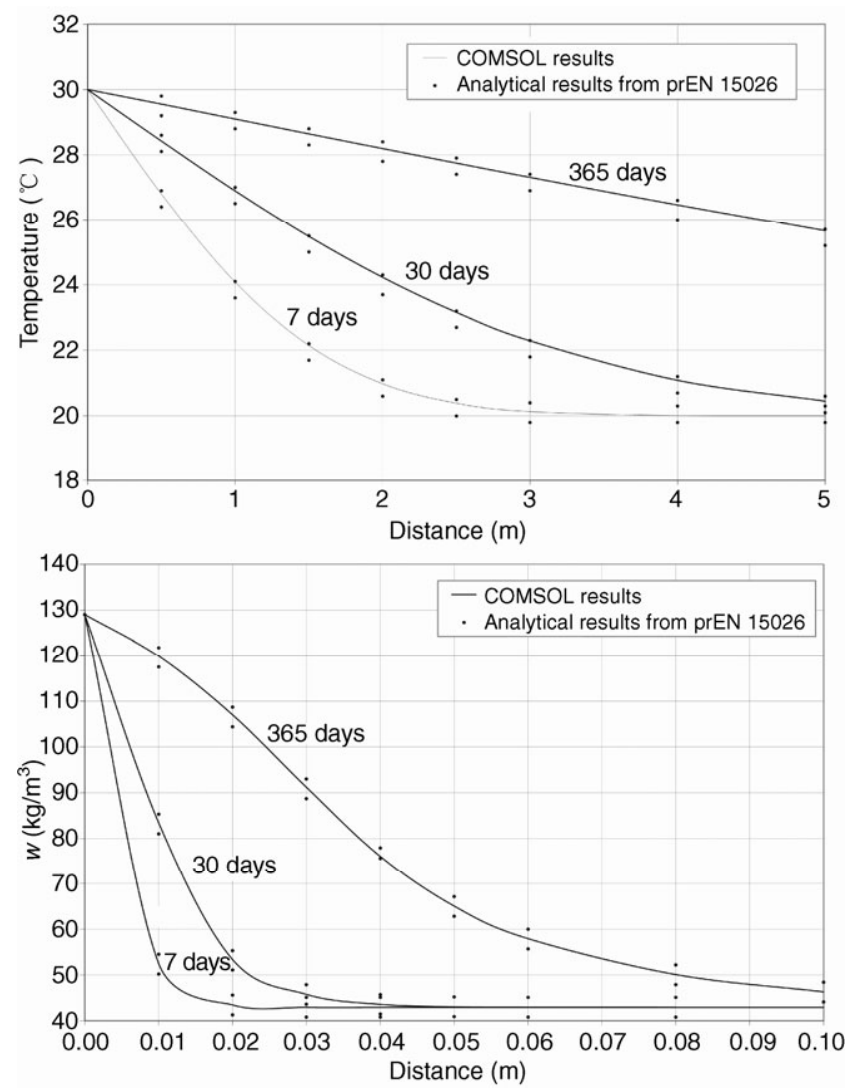

Fig. 2 The numerically simulated temperature and water content distribution profiles meeting the limits of validity stated by prEN15026 at 7 days, 30 days and 365 days
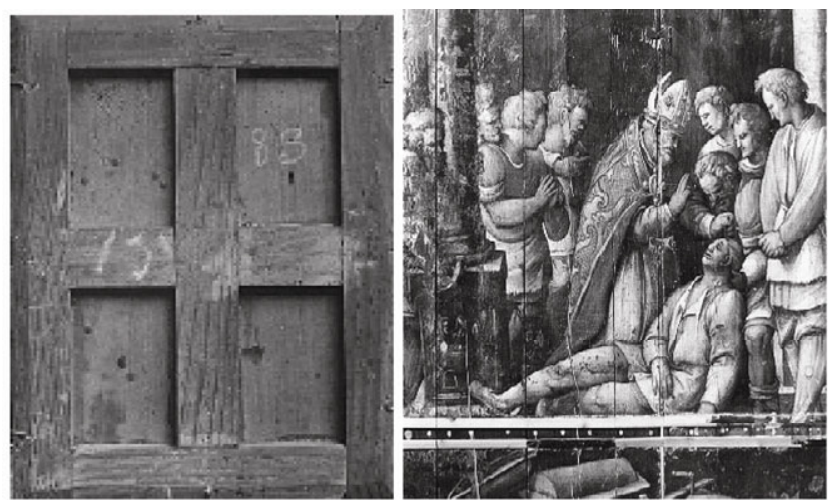

Fig. 3 Use of wood panels in historical paintings (Dardes and Rothe 1995) 
wood specimen to two cycles of 100 hours with relative humidity step variations between $47 \%$ and $35 \%$ under a constant temperature of $24^{\circ} \mathrm{C}$. Both experimental and numerical results of the dimensional response of a lime wood specimen under the test scenario are provided in the study. The numerical results presented in (Rachwal et al. 2010) were obtained using a model developed in a finite element method software package.

The hygrothermal material and mechanical properties of lime wood were determined experimentally by Rachwał et al. (2010) and were used as input in the HMSS model. Lime wood is treated as an orthotropic material in the model; as such, radial and tangential material and mechanical properties are included. Examples of input are the moisture content curve and moduli of elasticity are expanded in (Williams Portal 2011). It should be noted that the equilibrium moisture content (i.e. based on the re-adsorption curve) was used to define the moisture content in the lime wood panel in accordance to (Rachwał et al. 2010).

The numerical simulation results from the HMSS model were compared to both experimental and numerical results from the study as shown in Fig. 4 . The critical strain of lime wood was determined to be 0.002 (Rachwał et al. 2010) and the resulted strains were within this limit. The agreement between the HMSS numerical results and the experimental results from (Rachwał et al. 2010) is good. The observation of slight discrepancies could stem from the experimental uncertainty bounds existing during the determination of the hygrothermal properties of the wood specimen. Based on analysis, conducted by James (2010), of the hygrothermal response of different uncoated and coated gypsum boards during a step-varying moisture loading, hygrothermal numerical simulations were shown to be rather precise in this type of benchmark. Moreover, the precision of

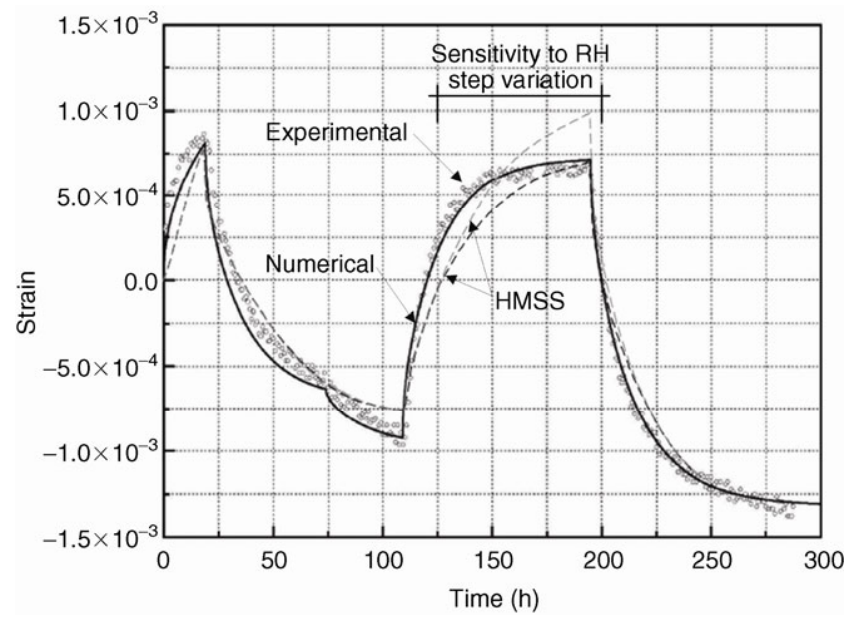

Fig. 4 Dimensional response in the tangential direction of a lime wood specimen to two variations of relative humidity between $47 \%$ and 35\%: experimental data (circles), study numerical results (solid line), HMSS numerical results (dashed lines) (Rachwał et al. 2010) experimental evaluation of the sorption isotherm and vapour permeability of the materials were decisive for the outcome of numerical validations, while the surface moisture transfer coefficient and hysteresis were of less importance.

In this case, comprehensive input data for experimental uncertainty bounds of sorption isotherms were regrettably not extractable from (Rachwal et al. 2010), which could explain the observed deviations in the HMSS numerical results for both steps. The information known from previous research includes: relative humidity ranging between $47 \%$ and $35 \%$ at estimated step variations. Therefore, only the sensitivity of the dimensional response to minimal dissimilarities in the prescribed relative humidity step variation is made evident by the two numerical simulation results from the HMSS model particularly between 125 and 200 hours. Accordingly, additional refinement of the hygrothermal properties would be necessary to increase the precision of the HMSS numerical simulation results. Nevertheless, the HMSS model simulates the expected response behaviour of lime wood within its critical strain limit and is thus considered an adequate predictive tool.

\section{Case study}

A case study is used in order to demonstrate the applicability of the two-dimensional HMSS model to historical buildings. The chosen case study involves the heritage site of the Castle of Gaasbeek (www.kasteelvangaasbeek.be) located in Gaasbeek, Belgium, illustrated in Fig. 5. This building was originally constructed in $1240 \mathrm{AD}$ and has been reconstructed and modified various times since then. This castle functions principally as a museum, but also contains offices and storage rooms. The castle's interior contains a multitude of art treasures, such as historic oil paintings and wooden furniture and statues, which can alone be considered as a cultural heritage site (Huijbregts et al. 2011).

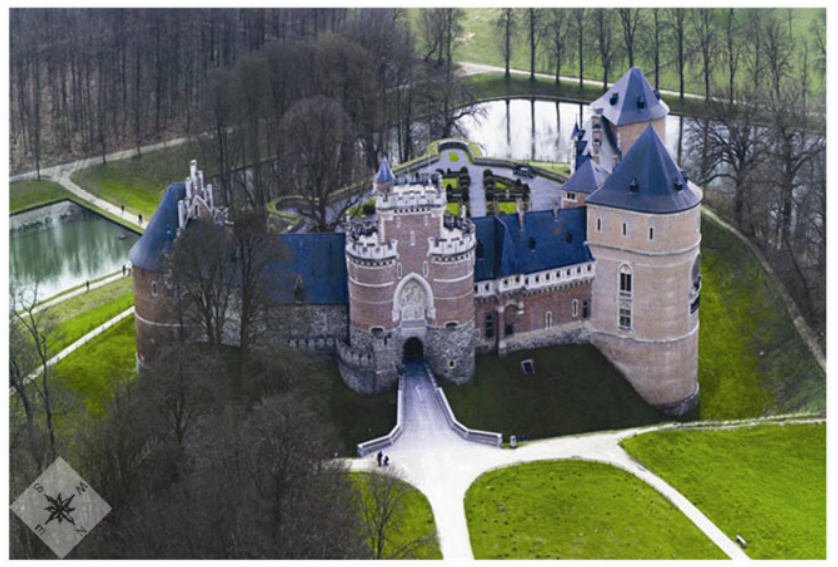

Fig. 5 Overview of the north east elevation of the Castle of Gaasbeek (www.kasteelvangaasbeek.be) 


\subsection{Climate data}

The indoor climate data includes annual temperature and relative humidity measurements from the Castle of Gaasbeek. The data was retrieved from Eindhoven University of Technology's online database entitled Physics of Monuments (Martens 2012). More specifically, the data used in this numerical simulation was obtained from a data collector situated at the interior of the first floor adjacent to the south east perimeter wall as per Fig. 6 .

The outdoor climate data used in this case study was obtained from the climate model REMO provided by the Max Planck Institute in Hamburg, Germany (Jacob and Podzun 1997). This climate data spans over a period from 1950 to 2099 and is the average data for several areas near De Bilt, the Netherlands (Huijbregts et al. 2012).

As previously discussed in Section 4.1, realistic boundary conditions exist and are important to include in modeling. In this case study, convective heat and moisture boundary conditions are included for both indoor and outdoor environments. In Section 5.4, rain is included as an additional moisture source (i.e. rain) in the HM model; the sensitivity of this parameter is analysed. Furthermore, the climate data prescribed in the simulations spanned over a time series of one year corresponding to January 2010 to January 2011. The prescribed temperature and relative humidity boundary conditions are presented in Fig. 7. This short time series was prescribed for the sole purpose of demonstrating the functionality and potential use of the HMSS model. In order to be able to derive valuable conclusions regarding long-term degradation processes in the materials, simulations spanning over larger time series are necessary.

\subsection{Modeling}

A two-dimensional HMSS model of a lime wood panel painting fixed to an exterior brick wall is considered in order

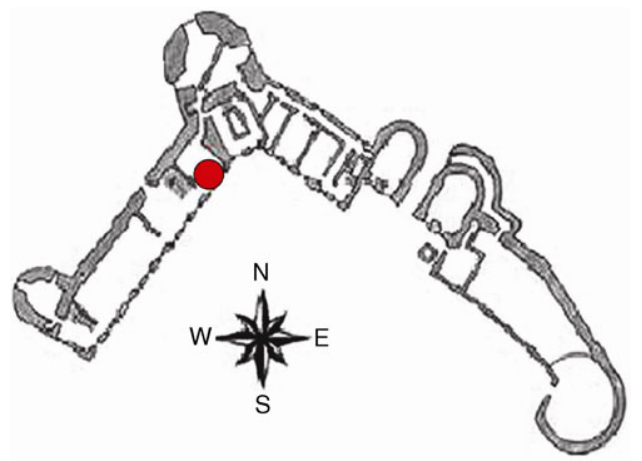

Fig. 6 Floor plan of the Castle of Gaasbeek and location of the data collector (red circle) (Huijbregts et al. 2011)
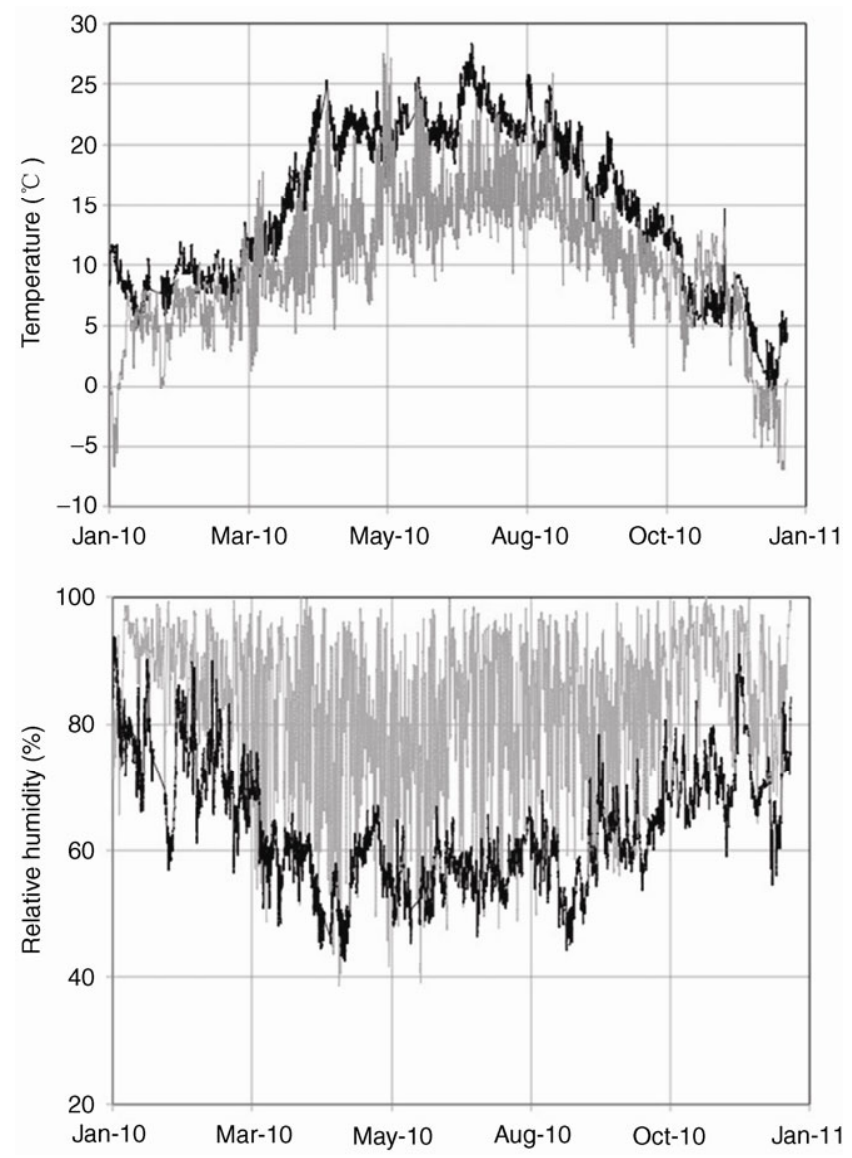

Fig. 7 Prescribed temperature $\left({ }^{\circ} \mathrm{C}\right)$ and relative humidity (\%) boundary conditions: outdoor (grey) and indoor (black)

to observe the interaction of artefacts with their surrounding building assemblies in historic buildings. The exterior wall assembly was defined to consist of two layers: historical solid brick $(380 \mathrm{~mm})$ and cement plaster $(20 \mathrm{~mm})$. The material properties of brick and plaster were obtained from the Fraunhofer-Institut for Bauphysik (IBP) Holzkirchen material database (IBP 2010). The panel painting consists of a lime wood panel $(10 \mathrm{~mm})$ with gesso finish in accordance with typical dimensions and material properties derived from the study by Rachwał (2010). A general overview of the geometry for the two-dimensional HMSS model is shown in Fig. 8.

A wooden panel painting is commonly placed with a certain distance from the wall. The air gap that is created thereby serves as a capillary break, which protects the wood from capillary transported moisture in the brick wall. A proper air circulation through the air gap cannot be ensured unless it is kept adequately accessible for air movement (Mortensen et al. 2008). Common supporting systems of panels are constructed of densely placed crossbars (i.e. Marcon et al. 2012; Mazzanti et al. 2012), which prevent a proper air circulation. As there is a large uncertainty about 


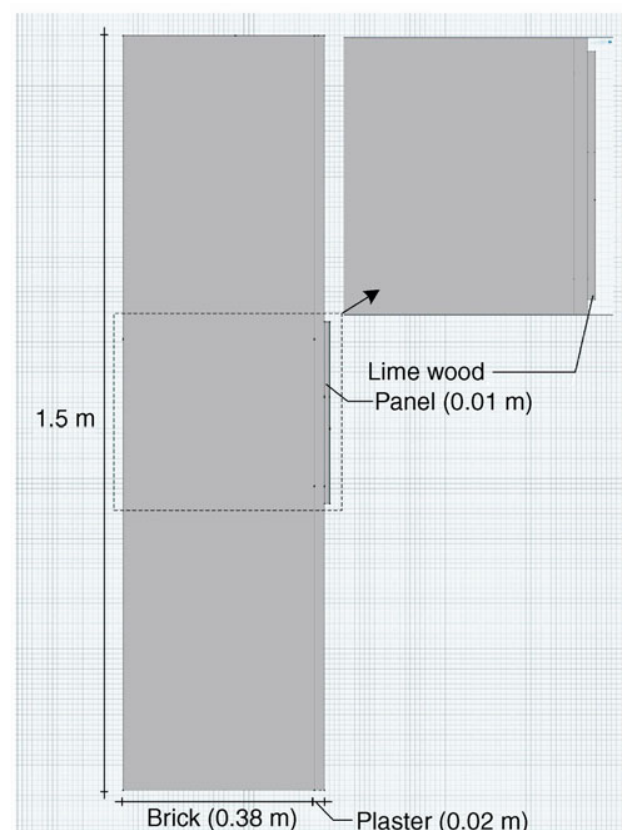

Fig. 8 Overview of the geometry $(\mathrm{m})$ for the two-dimensional HMSS model in COMSOL

the rate of air circulation through the gap in the studied case, the resistance of the gap is neglected in the simulations. In this way, the hygrothermal conditions at the interface between the wall and the panel are the most severe ones in terms of possible hygrothermal loads from the wall to the panel.

The numerical simulation was carried out according to Fig. 1. The HM process indicated by Zone 1 was computed for both the exterior wall and the panel painting according to the prescribed climatic boundary conditions. Subsequently, only the simulated HM results for the lime wood panel painting are coupled to the HMSS process indicated by Zone 2. In this case study, it was only of particular interest to observe the hygrothermal-induced mechanical behaviour of the panel painting.

\subsection{HMSS model results}

The numerical simulation was computed for a time period of one year for the purpose of demonstrating the possible numerical results that can be yielded from the HMSS model, as aforementioned in Section 5.1. It should be noted that a longer time period is necessary in order to yield results that can be used to evaluate the long-term effects of material degradation. Figures 9 and 10 depict the numerical simulation results for the temperature and moisture distributions in the geometry. The magnified deformation of the panel painting is also shown in these figures with a scale factor of 62.5 .

Furthermore, the displacement field results occurring at the centre of the panel painting in the tangential direction

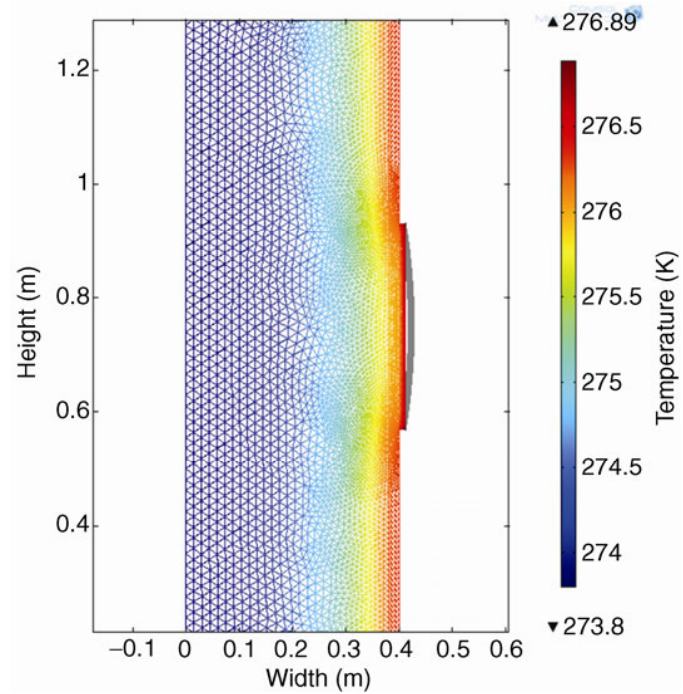

Fig. 9 Numerical simulation results for the temperature distribution $(\mathrm{K})$ in the exterior wall and panel painting $(\mathrm{m})$ after one year

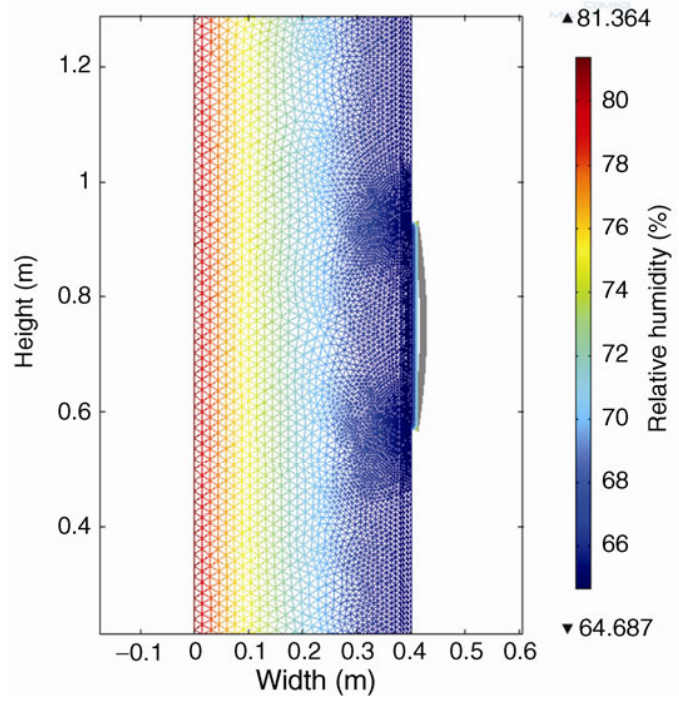

Fig. 10 Numerical simulation results for the relative humidity distribution (\%) in the exterior wall and panel painting (m) after one year

( $y$-direction) are summarized in Fig. 11. The displacement field is a measurement of the expansion and contraction of the wood panel as a result of its exposure to changing climate boundary conditions. The dimensional response of the panel painting takes place concurrently with the variations in the boundary conditions, particularly concerning differences between the relative humidity inside the panel and at the interior of the panel. The largest displacement is observed at the start of the simulation, when a relatively dry panel (with relative humidity of 60\%) is introduced in a more humid environment of the museum (almost 90\%). It should be noted that the relative humidity is a good indication of the moisture content inside the panel, as there are no larger temperature differences between the panel 


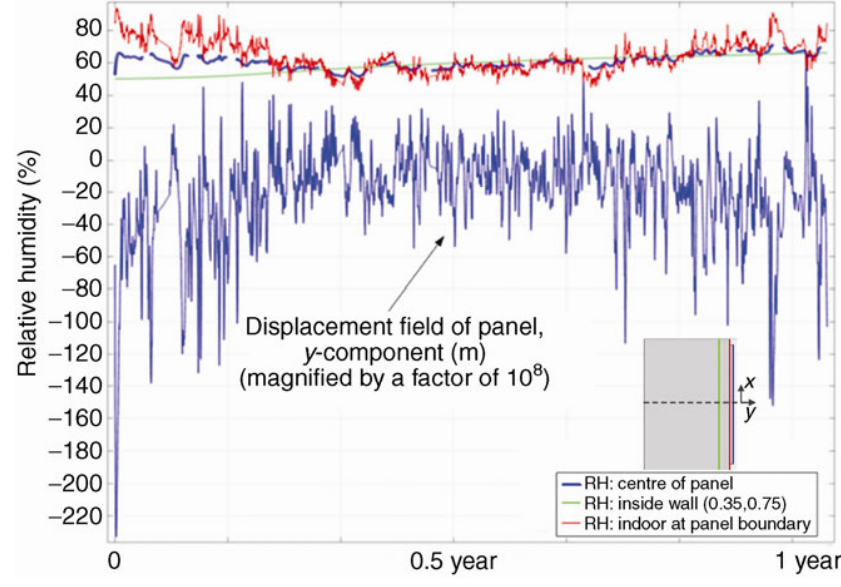

Fig. 11 Numerical simulation results of the dimensional response in the tangential direction ( $y$-direction) at the centre of the panel painting $(\mathrm{m})$ (magnified by a factor of $10^{8}$ ) compared to the relative humidity at the centre of the panel, inside the wall $(0.35,0.75)$ and at the indoor panel boundary, after one year

and the indoor environment as previously shown in Fig. 9. The initial displacement of the panel largely decreases during the warmer period of the year, because the relative humidity in the museum is approximately the same as inside the panel. At the end of the year, when the climate in the museum becomes more humid again, the displacement of the panel increases. There is less displacement in the panel at the end of the year in comparison to that at the start of the simulation due to increased moisture content inside the panel. The wetting of the panel occurs from two sides-from the interior, but also from the adjacent side of the brick wall, whose moisture content also increases over the course of the year as shown in Fig. 13 (see Section 5.4).

\subsection{Sensitivity analysis}

In the case study presented in Section 5, the impact of rain on the wall was neglected because the existing wall was found in a rather sheltered position with respect to rain. The impact of rain as an external boundary condition was thus investigated in this sensitivity analysis. The prescribed rain data was obtained from (Jacob and Podzun 1997) and spaned over a period of one year corresponding to January 2010 to January 2011.

In this analysis, possible uncertainties can be caused by:

- Possible rain intensity differences during the hour could cause erroneous results, as hourly mean values for the rain intensities are used in the analysis. For example, during an hour of heavy rain, it is possible that the rain event is realistically occurring during a short period of time (i.e. 10 minutes). In this case, the runoff is expected to be much higher in comparison to a rain event that is more evenly distributed during the hour. Consequently, the amount of rain entering the exterior boundary of the construction is much lower, such that there is uncertainty in terms of the fraction of moisture entering the material during each hour of a rain event. As such, high intensity will cause a high level of uncertainty. More precise simulations could be easily achieved by using smaller time steps for the reading of climate data, but at the expense of total simulation time, see Fig. 12.

- Besides the temporal resolution, it was observed that the space resolution of the numerical grid also influences the results. For highly capillary active materials such as brick, a coarse numerical mesh (478 triangular elements) may introduce an artificial resistance to the capillary transported moisture between the elements in a wet region (i.e. exposed to rain) and the elements in a dry region (i.e. inside the wall). A similar problem was observed with other numerical techniques such as finite volumes (Sasic Kalagasidis et al. 2004). A finer numerical mesh (909 triangular elements) gives more moisture in the wall and a smoother redistribution of moisture across the wall after each rain event, see Fig. 12. The difference between the total moisture content inside the brick wall in the case study is, after one year, approximately $10 \%$, see Fig. 13 . Similarly, a finer mesh leads to better results but at the expense of total simulation time.

- Numerical errors could also be the cause of uncertainties in the analysis as shown in Fig. 14. The differences between the two simulations, namely coarse grid (maximum time

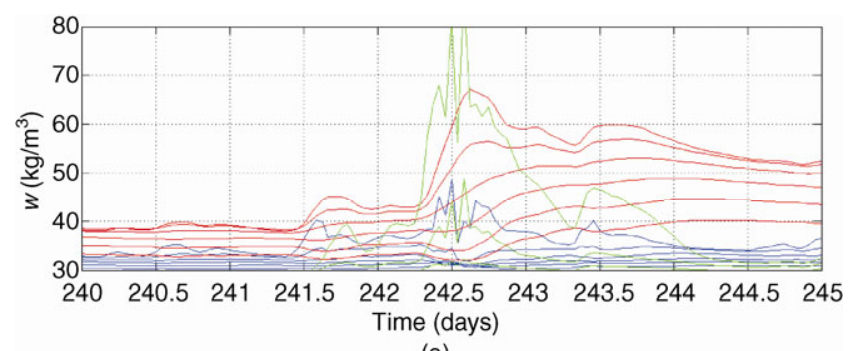

(a)

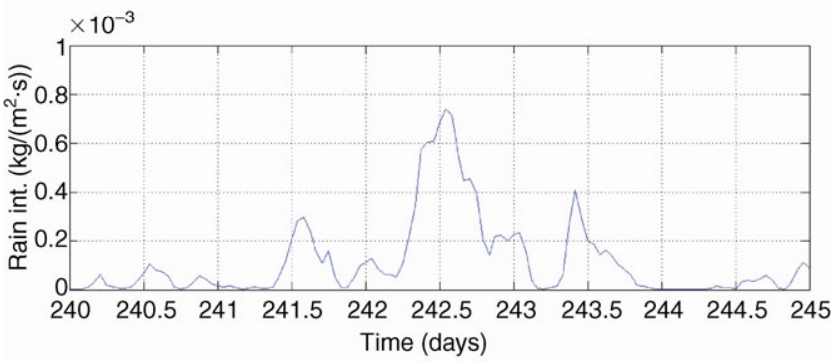

(b)

Fig. 12 The moisture distribution through the thickness of the wall $(x=0,0.01,0.02,0.03,0.04,0.05 \mathrm{~m})$ at a given time (days) after a heavy rain event. (a) Blue, standard grid and maximum time step of $1800 \mathrm{~s}$; red, fine grid and maximum time step of $600 \mathrm{~s}$; green, coarse grid and maximum time step of 1800 s. (b) Rain intensity 


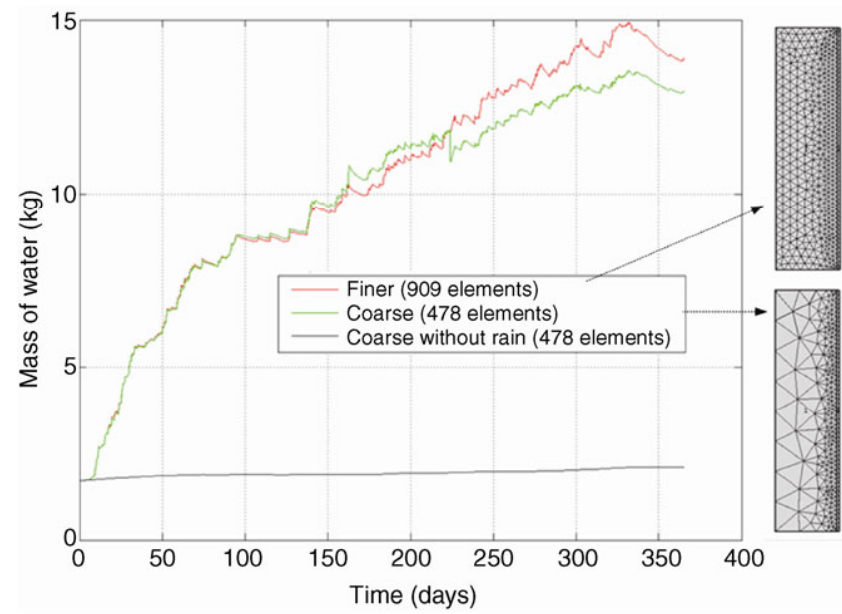

Fig. 13 The total moisture content of the outer wall. Red: fine grid (909 triangular elements and maximum time step of $600 \mathrm{~s}$ ); green: coarse grid (478 triangular elements and maximum time step of 1800 s); black: coarse grid without rain (478 triangular elements and maximum time step of $1800 \mathrm{~s}$ )

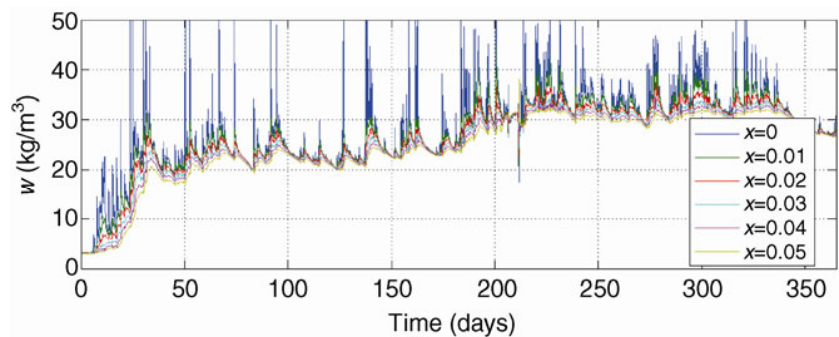

(a)

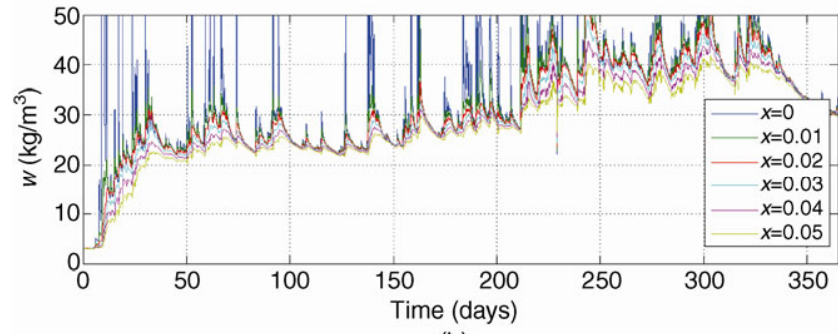

(b)

Fig. 14 Moisture contents at different positions in the material $(\mathrm{mm})$. (a) Coarse grid (478 triangular elements and maximum time step of $1800 \mathrm{~s}$ ); (b) fine grid (909 triangular elements and maximum time step of $600 \mathrm{~s}$ )

step of $1800 \mathrm{~s}$ ) and finer grid (maximum time step of $600 \mathrm{~s}$ ), are influenced by the observed behaviour at the surface $(x=0)$. The differences in spikes at the surface occur due to numerical errors and grid difference which, in turn, cause numerical uncertainties at the surface.

- Although the modelling of hysteresis effects has not been in the scope of this work, it could have implications on the HMSS response of the wood panel. It is estimated that the inclusion of adsorption and desorption isotherms is rather straight-forward and could be readily achieved in the chosen modelling environment using an additional calculation routine.

\section{Conclusions}

One- and two-dimensional transient coupled heat and moisture induced mechanical stress and strain (HMSS) models were developed in COMSOL Multiphysics. Onedimensional transient decoupled and coupled heat and moisture (HM) models were implemented and verified by means of analytical solutions and benchmarks. The heat and moisture induced stress and strain mechanisms were successively included into the verified HM model to formulate a two-dimensional HMSS transient model. The latter model was validated through experimental and numerical results derived from a study evaluating the response of lime wood supports in historical panel paintings subjected to changing climate conditions. The verified HMSS model was lastly tailored to a case study in order to demonstrate the applicability of the model to the interaction of artefacts and building assemblies of historical buildings.

The following conclusions can be summarized for this paper:

- The coupled HM model is a valid predictive tool to investigate the impact of a change in climatic conditions on building assemblies and artefacts.

- The HMSS model is an adequate predictive tool to determine possible damage-related processes in building assemblies and artefacts.

- The verification of the HMSS model is based on a specific case study and the mechanical behaviour of lime wood, which in turn signifies that additional verification using other desired materials may need to be executed.

\section{Recommendations}

The recommendations for future applications and developments related to this paper include the following:

- The evaluation and prediction of the effects of climate change on the HMSS in building assemblies and artefacts found in historical buildings.

- The establishment of guidelines for future indoor climate demands based on the findings to prevent predicted damages to building assemblies and artefacts found in historical buildings.

- The investigation of the cause of damage-related processes in building assemblies and artefacts of heritage sites.

\section{References}

Bratasza L, Harris I, Lasyk L, Łukomski M, Kozłowski R (2012). Future climate-induced pressures on painted wood. Journal of Cultural Heritage, 13: $365-370$.

Burke S (2009). Building Physics Tools: Needs, Use and the Lack of Use in the Building Process. Modeling Non-isothermal Moisture Flow and Frost Penetration. PhD Thesis, Lund University of Technology, Sweden. 
Castellini P, Esposito E, Marchetti B, Paone N, Tomasini EP (2003). New applications of Scanning Laser Doppler Vibrometry (SLDV) to non-destructive diagnostics of artworks: Mosaics, ceramics, inlaid wood and easel painting. Journal of Cultural Heritage, 4: $321-329$.

Claesson J (2002). Boltzmann solution of coupled nonlinear equations for moisture content $\mathrm{w}(\mathrm{s})$ and temperature $\mathrm{T}(\mathrm{s}), \mathrm{s}=\mathrm{x} / \operatorname{root}(4 \mathrm{t})$. Benchmark test I, Chalmers University of Technology, Sweden.

COMSOL (2010). COMSOL Multiphysics Version 4.1. COMSOL AB.

Dardes K, Rothe A (1995). The Structural Conservation of Panel Paintings. In: Proceedings of a symposium at the J. Paul Getty Museum, Los Angeles US. Available: http://www.getty.edu/ conservation/publications/pdf_publications/panelpaintings3.pdf.

Dionisi Vici P, Mazzanti P, Uzielli L (2006). Mechanical response of wooden boards subjected to humidity step variations: Climatic chamber measurements and fitted mathematical models. Journal of Cultural Heritage, 7: 37 - 48.

Eschenauer H, Olhoff N, Schnell W (1997). Applied Structural Mechanics. Berlin: Springer-Verlag.

Fraunhofer Institut für Bauphysik (IBP) Holzkirchen (2010). WUFI 2D Version 3.3.

Hagentoft C-E (1998). The impact of heat, air and moisture transport on energy demand and durability, Final report. International Energy Agency Annex 24. Task 5: Performances and Practice.

Hagentoft C-E (2011). Introduction to Building Physics. Lund, Sweden: Studentlitteratur.

Hagentoft C-E (2002). HAMSTAD—WP2 Modeling, Report R-02:9. Chalmers University of Technology, Sweden.

Huijbregts Z, Kramer RP, Martens MHJ, van Schijndel AWM, Schellen HL (2012). A proposed method to assess the damage risk of future climate change to museum objects in historic buildings. Building and Environment, 55: 43 - 56.

Huijbregts Z, Kramer RP, van Schijndel AWM, Schellen HL (2011). Computational modeling of the impact of climate change on the indoor environment of a historic building in the Netherlands. In: Proceedings of 9th Nordic Symposium on Building Physics, Tampere, Finland, pp. $833-840$.

International Institute of Conservation (ICA) (2010). The Plus/Minus Dilemma: The Way Forward in Environmental GuidelinesEdited transcription of the roundtable. Available: http://www.iiconservation.org/dialogues/Plus_Minus_trans.pdf.

Jacob D, Podzun R (1997). Sensitivity studies with the regional climate model REMO. Meteorology Atmospheric Physics, 63: 119 - 129.

Jakieła S, Bratasz Ł, Kozlowski R (2008). Numerical modeling of moisture movement and related stress field in lime wood subjected to changing climate conditions. Wood Science and Technology, 42: $21-37$.

James C, Simonson CJ, Talukdar P, Roels S (2010). Numerical and experimental data set for benchmarking hygroscopic buffering models. International Journal of Heat and Mass Transfer, 53: 3638 - 3654 .

Janssen H (2011). Thermal diffusion of water vapour in porous materials: Fact or fiction? International Journal of Heat and Mass Transfer, 54: 1548 - 1562 .

Kowalkski SJ, Rybicki A (2004). Drying induced stresses in a swelling porous wall. Journal of Transport in Porous Media, 57: 35 - 48.

Kowalski SJ, Smoczkiewicz-Wojciechowska A (2007). Stresses in dried wood. Modeling and experimental identification. Transport in Porous Media, 66: 145 - 158.
Łukomski M (2012). Painted wood. What makes the paint crack? Journal of Cultural Heritage, 13(Supplement): s90 - s94.

Marcon B, Mazzanti P, Uzielli L, Cocchi L, Dureisseix D, Gril J (2012). Mechanical study of a support system for cupping control of panel paintings combining crossbars and springs. Journal of Cultural Heritage, 13(Supplement): s109 - s117.

Martens MHJ (2012). Climate Risk Assessment in Museums. PhD Thesis, Eindhoven University of Technology, the Netherlands.

Mazzanti P, Togni M, Uzielli L (2012). Drying shrinkage and mechanical properties of poplar wood (Populusalba L.) across the grain. Journal of Cultural Heritage, 13(Supplement): s85 - s89.

Mihoubi D, Bellagi A (2009). Stress generated during drying of saturated porous media. Journal of Transport in Porous Media, 80: 519 - 536.

Mirianon F, Fortino S, Toratti T. (2008). A method to model wood by using ABAQUS finite element software. Part 1. Constitutive model and computational details. VTT Technical Research Centre of Finland, VTT Publications 687.

Mortensen LH, Rode C, Peuhkuri R (2008). Investigation of airflow patterns in a microclimate by particle image velocimetry (PIV). Building and Environment, 43: 1929 - 1938.

Nicolai A, Grunewald J, Zhang JS. (2007). Salt mixture transport and phase transitions, Modeling and numerical solution in Delphin 5. In: Proceedings of 12th Symposium for Building Physics, Dresden, Germany.

Ottosen N, Petersson H (1992). Introduction to the Finite Element Method. Harlow, UK: Pearson/Prentice Hall.

prEN 15026 (2004). European Provisional Standard prEN 15026: Hygrothermal Performance of Building Components and Building Elements-Assessment of Moisture Transfer by Numerical Simulation.

Rachwał B, Bratasz Ł, Łukomski M, Kozłowski R (2010). Response of wood supports in panel paintings subjected to changing climate conditions. Strain, 48: 366 - 374 .

Sasic Kalagasidis A, Bednar T. Hagentoft C-E (2004). The evaluation of the interface moisture conductivity between control volumes: Comparison between linear, harmonic and integral averaging. In: Proceedings of 9th International Conference on Performance of the Exterior Envelopes of Whole Buildings, Clearwater Beach.

Schellen HL, van Schijndel AWM (2011). Setpoint control for air heating in a church to minimize moisture related mechanical stress in wooden interior parts. Building Simulation, 4: $79-86$.

van Schijndel AWM (2011). Multiphysics modeling of building physical constructions. Building Simulation, 4: $49-60$.

Segerholm I (2007). Moisture Transport Processes in Scots PineAnomalous Capillary Suction, Nonisothermal Diffusion. PhD Thesis, Chalmers University of Technology, Sweden.

Sevilgen G, Kilic M (2011). Numerical analysis of air flow, heat transfer, moisture transport and thermal comfort in a room heated by two-panel radiators. Energy and Buildings, 43: 137 - 146.

Williams Portal N (2011). Evaluation of heat and moisture induced stress and strain of historic building materials and artefacts. Master Thesis, Chalmers University of Technology, Sweden.

Williams Portal N, Sasic Kalagasidis A, van Schijndel AWM (2011). Simulation of heat and moisture induced stress and strain of historic building materials. In: Proceedings of 12th IBPSA International Conference, Sydney, Australia.

Woloszyn M, Rode C (2008). Tools for performance simulation of heat, air and moisture conditions of whole buildings. Building Simulation, 1: 5- 24 . 